\title{
Reasons (not) to Spend a Few Billions More on EHRs: How Human Factors Research Can Help
}

\author{
G. Declerck, X. Aimé, Section Editors for the IMIA Yearbook Section on Human Factors and \\ Organizational lssues
}

INSERM, U1142, LIMICS, F-75006, Paris, France

Sorbonne Universités, UPMC Univ Paris 06, UMR S 1142, LIMICS, F-75006, Paris, France

Université Paris 13, Sorbonne Paris Cité, LIMICS, (UMR_S 1142), F-93430, Villetaneuse, France

\begin{abstract}
Summary
Objectives: To select best medical informatics research works published in 2013 on electronic health record (EHR) adoption, design, and impact, from the perspective of human factors and organizational issues (HFOI).

Methods: We selected 2,764 papers by querying PubMed (Mesh and TIAB) as well as using a manual search. Papers were evaluated based on pre-defined exclusion and inclusion criteria from their title, keywords, and abstract to select 15 candidate best papers, finally reviewed by 4 external reviewers using a standard evaluation grid.

Results: Five papers were selected as best papers to illustrate how human factors approaches can improve EHR adoption and design. Among other contributions, these works: (i) make use of the observational and analysis methodologies of social and cognitive sciences to understand clinicians' attitudes towards EHRS, EHR use patterns, and impact on care processes, workflows, information exchange, and coordination of care; (ii) take into account macro- (environmental) and meso- (organizational) level factors to analyze EHR adoption or lack thereof; (iii) highlight the need for qualitative studies to analyze the unexpected side effects of EHRs on cognitive and work processes as well as the persistent use of paper.

Conclusion: Selected papers tend to demonstrate that HFOI approaches and methodologies are essential to bridge the gap between EHR systems and end users, and to reduce regularly reported adoption failures and unexpected consequences.
\end{abstract}

\section{Keywords}

Medical informatics, human factors, organizational issues, electronic health record, health information technology adoption

Yearb Med Inform 2014:90-6

http://dx.doi.org/10.15265/IY-2014-0033

Published online August 15, 2014

\section{Introduction}

Despite substantial financial incentives from governments, electronic health records (EHRs) are still underused and the adoption process is much slower and more prone to healthcare professionals' (HPs) resistance than expected. In the US, the $\$ 20$ billion invested through the Health Information Technology on Economic and Clinical Health (HITECH) Act led undoubtedly to some improvement of the EHR implementation rate [1], but most criteria for meaningful use are still not met [2-4]. In European countries such as France, the government invested more than half a billion Euros between 2004 and 2011 for the deployment of a personal health record that would be connected to hospital EHRs [5], with (at best) disappointing results, and plans to invest 400 million Euros between 2013 and 2017 for the promotion of EHRs in hospitals through the "Digital Hospital Program" [6]. In addition, the positive impact of EHRs on healthcare quality and safety or on cost reduction and optimization has still to be demonstrated [7]. DesRoches et al. [8] found a striking lack of relationship between the adoption of EHR systems in hospitals and the quality and efficiency of care, which suggests that adoption (whatever this may mean) is not sufficient to drive improvements of care processes. How can these difficulties be explained?

The collective notion we have of EHRs (and what EHRs must be and offer) undoubtedly shares some responsibility in this situation. One of the most widespread - but largely unquestioned - assumptions by health authorities in regards to EHRs is that EHR adoption "is essential for the transformation of the current [...] healthcare system into one that is more efficient, is safer, and consistently delivers high-quality care" [9]. One reason for such a deep trust in technology derives from the critical importance of integrity, reliability, and accuracy of information in the care process and the supposedly increased reliability of patient data encoded in electronic systems (as compared with paper) [10]. EHRs are often introduced to improve information exchange amongst providers [11], e.g. for ensuring a reliable information transfer during patient handovers, where safe practice and quality of care depend on the transmission of data [12].

However, while technology is undoubtedly necessary to improve healthcare, it is certainly not sufficient. Handovers cannot be reduced to information transfers. Focusing on criteria such as information completeness and information accuracy (sometimes characterized as an "info-centric" view of information transfer) is not enough. As Wong et al. [13] explain, "Information is only part of the problem and hence solely addressing that issue is only part of the solution". Based on in depth ethnographic case studies, Balka et al. [11] e.g. demonstrated that several aspects of clinical handovers, such as the contextual nature of information, ethical issues, and professional norms, must be addressed before the EHR-based patient handover can contribute to time efficiencies and error reduction.

More importantly, a growing body of observations suggests that the deployment of EHRs in workplaces creates more challenges than expected. A number of negative 
effects of EHRs and their implementation on the quality of care or other dimensions of the healthcare process have been documented. Several negative aspects of EHRs were highlighted in a RAND study [14] commissioned by the American Medical Association. Factors affecting physicians' professional satisfaction negatively include increased time required for data entry, mismatches between user interfaces and clinical workflow, interferences with physician-patient face-to-face conversation, lack of health information exchange, information overload, and deterioration of clinical documentation. Physicians agreed with the concept of EHRs and appreciated the new potentialities enabled by such tools like remote access to patient information. However, for many of them, current EHR systems significantly worsened their professional satisfaction in multiple ways.

Even the common idea that EHR systems ensure a (more) reliable (than paper) information encoding and transfer is not substantiated by real-world experience. As Beasley et al. [15] explain, "The emergence of EHR-related errors results in data being lost or incorrectly entered, displayed, or transmitted, leading to loss of information integrity". Electronic encoding of data also raises major challenges. Transfer of data from one care provider to another usually requires an understanding of the terminology used to encode the data and a translation or an interpretation taking into account the context of data collection. When encoded, the information is subject to "professional norms and standards which currently require each new provider to re-check this information" [11]. How to take these norms into account in EHR systems? Standardizing data architectures across provider groups is a solution, although flexible standardization approaches are necessary to enable individual fields to be tailored to local requirements [16].

Another basic positive effect expected from EHRs is the reduction of cognitive (and especially information) overload, which is a well-known challenge for healthcare practices and has implications on both physician performance and patient safety $[15,17]$. However, here again, it has been demonstrated that EHRs frequently increase physician cognitive demands [18] and may lead to decreased productivity [9]. Babbott

Table 1 Best paper selection of articles for the IMIA Yearbook of Medical Informatics 2014 in the section 'Human Factors and Organizational Issues'. The articles are listed in alphabetical order of the first author's surname.

Section

Human Factors and Organizational Issues

- Babbott S, Manwell LB, Brown R, Montague E, Williams E, Schwartz M, Hess E, Linzer M. Electronic medical records and physician stress in primary care: results from the MEMO Study. J Am Med Inform Assoc 2014 Feb;21(el):e100-6.

- Flanagan ME, Saleem JJ, Millitello LG, Russ AL, Doebbeling BN. Paper- and computer-based workarounds to electronic health record use at three benchmark institutions. J Am Med Inform Assoc 2013 Jun;20(el):e59-66.

- Hilligoss B, Zheng K. Chart biopsy: an emerging medical practice enabled by electronic health records and its impacts on emergency department-inpatient admission handoffs. J Am Med Inform Assoc 2013 Mar-Apr;20(2):260-7.

- Lanham HJ, Sittig DF, Leykum LK, Parchman ML, Pugh JA, McDaniel RR. Understanding differences in electronic health record (EHR) use: linking individual physicians' perceptions of uncertainty and EHR use patterns in ambulatory care. J Am Med Inform Assoc 2014 Jan 1;21(1):73-81.

- Smith SW, Koppel R. Healthcare information technology's relativity problems: a typology of how patients' physical reality, clinicians' mental models, and healthcare information technology differ. J Am Med Inform Assoc 2014 Jan 1;21(1):117-31.

et al. [20] also report that EHRs can increase cognitive workload especially during the process of practice transformation, which happens in the first implementation period, because HPs use a hybrid system composed of paper and electronic records, "each requiring different processes and cognitive demands". This hybrid system can "lead to problems with coordinating care, and concerns about tracking orders and summaries".

Last but not least, the deployment of an EHR system in a medical institution does not imply that it will be used, and use does not mean it is used as expected. Most deployed EHRs are not used or when used, only their most basic functionalities are used. As highlighted by Nambisan et al. [2], "approximately $19 \%$ of [EHRs] are uninstalled after implementation, and approximately $30 \%$ are not used to their full potential by the care staff." Workarounds (whether paper-based or electronic) to avoid using EHRs are also frequently observed [21]. "Shadow charts" based on hand-written notes are reported to be commonly used in many institutions to track patient progress or as memory aids for handoff, and are generally believed to be more accurate and up-to-date than the patient information provided in EHRs [22, 23].

To explain the disappointing results, a growing number of authors point to the lack of understanding of human and socio-organizational factors affecting EHR adoption as well as methodological insufficiencies [2, $24,25]$. What is currently lacking is a robust interdisciplinary research framework inte- grating cognitive and social sciences. Several reasons explain why such a framework would be relevant for overcoming the present EHR adoption "crisis". First, current research on EHR adoption suffers from what Nambisan et al. [2] call a "silo" effect: factors implied at the macro- (environmental), meso- (organizational) and micro- (individual) levels are studied usually in isolation. For instance, works dealing with individual level factors (for example psychological and cultural factors of physician resistance to technology) tend to ignore the economic perspective and institutional focus. An integrative understanding of EHR adoption is needed that incorporates multiple levels of analysis, and is capable of considering interactions and synergy effects between the factors implied at different levels. Complementary theoretical perspectives, particularly from human behavior, sociocultural sciences, or systems theory, are necessary to understand the impediments of EHR adoption fully [2].

Another reason why human factors approaches are relevant for current EHR research is that observational and analysis methodologies from social and cognitive sciences can help to elucidate concrete work practices of HPs that are essential for the care process but tend to be invisible and poorly accounted for by system designers [11]. These methods are also essential to objectify lacking functionality (or functionality that is not wanted, disrupting or has a negative impact on the work process) in deployed EHR systems determining why users reject them 
and prefer to use substitutes such as "ghost charts" $[21,22,23]$. Inevitably people will develop workarounds to complement the perceived limitations of the existing systems. As indicated by Mathews and Pronovost [26], "clinicians often feel burdened rather than supported by technology, [...] as a result, workarounds are common and solutions meant to help often directly conflict with one another". Studying these workaround strategies with adapted observational methods such as the ethno-methodological approach [27] is essential to determine how current EHR systems should evolve to better respond to expectations and needs of end users. Observational methods can also anticipate and assess EHR impact on behavior or practice, e.g. through the formalization of workflow variations across healthcare settings [28].

How HPs perceive EHRs is of course another key issue to consider. Human factors and cognitive or social sciences stress that a successful health information technology (HIT) must scaffold cognitive activities and work practices, facilitate and streamline what the physician must accomplish (generally by himself), and should not enslave the physician to computational functions and information requirements, which may result in mechanization of medical practices. The first chatbots (i.e. conversational agents) developed by Artificial Intelligence researchers required the user to speak a perfectly articulated academic English very slowly and distinctly. EHRs should not have these types of restrictions. The fact that EHR systems require structured data input (e.g. from standard biomedical terminologies) and tend to minimize free text (which cannot be computed easily) is evidently a challenge to their use.

EHR systems function also to rationalize, document, and control work practices (for example coding of medical treatment for hospitals for reimbursement by the social security system in most countries, e.g. in the US Medicare program). It is pointless to demonize these aspects of EHRs. However, the problem arises when bureaucratic functions interfere with, mask, or obstruct what is the primary or the perceived primary purpose of EHRs: To improve the care process. EHRs are frequently perceived by HPs as tools foremost dedicated to documentation and billing purposes, rather than as a cognitive aid for activities such as prospective memory and coordination [21]. To change HPs' perception of EHR systems as tools providing cognitive scaffolding, rather than tools for bureaucratic control remains a challenge for the design of future EHRs [21]. To achieve this goal requires emphasizing the benefits of EHRs in the daily practice, i.e. demonstrating to HPs that EHRs have become an indispensable tool for providing better care with less effort [28].

To date, most HIT designers remain trapped in technocentrism: to build a suitable EHR system, developers must design more than the tool itself, but must focus on the whole system that embeds the tool. EHRs are a part of a whole system. They will achieve adoption only if their properties are compatible with the properties of the other components of the whole. Interoperability is an obvious example. Ethical and legal issues associated with data privacy are another. EHR designers must realize that the EHR is not an isolated device with functionalities, but must conceptualize improving practices through technology. Cognitive science approaches that envision humans and technological devices as dependent components and explore a single coordinated ensemble are needed. The study of artefacts not as objects, but as systems that shape cognition and collaboration, is valuable. Noteworthy tools are:

a. Sociotechnical [29] and user-centered design [30] approaches;

b. Distributed cognition framework [3135], which moves the unit of analysis from the brain to the overall system possibly comprising several individuals exchanging information through several communication channels and relying on artifacts and environmental structures to achieve these tasks;

c. Situated, externalist, and enactivist approaches to cognition [36-40], which insist on the role played by the body, the tools, and the interactions with the environment in cognitive performances;

d. Cognitive technology studies, which are to date fairly unknown but may prove important to understand why HPs frequently disregard electronic tools, and prefer instead the paper sheets. Cognitive technology studies - based on the original work of the anthropologist Jack Goody [41] - analyze the cognitive properties of symbolic technologies, i.e. technologies to store and communicate information. Typically, writing enables a (quasi-)permanent encoding of the message, without alteration of its content, unlike the oral communication channel. The ubiquity of the electronic document, i.e. the possibility one could access and update it from several places simultaneously, is one of those remarkable cognitive properties. This contrasts with the "uniquity" of the paper document, which exists as one single instance at one single place at a time. But ubiquity is not always desirable when dealing with patient data [42].

e. Lastly, the work of Bertrand Gille [43] on technical systems is also a valuable resource to build a holistic perspective of the different social sub-systems that are implied in EHR adoption.

One lesson to be learnt from current research on EHR adoption is that EHRs have become indispensable but at the same time remain insufferable. Most EHR systems are not yet usable and may be more of a burden than a support for practitioners. However, EHRs are something healthcare institutions must eventually adopt: the degree of computerization in most developed countries makes this adoption unavoidable. The question is: what is required to design a whole macrocosm EHR system that would be compatible with all other systems involved? The 2014 selection of best papers for the Human Factors and Organizational Issues section of the Yearbook of Medical Informatics was compiled to address the different issues.

\section{Paper Selection Method}

Two PubMed queries, one on Mesh terms, the other on TIAB terms, were built, focusing on keywords related to HFOI. A manual search of seven highly rated medical informatics journals based on [44] completed the queries. Using PubMed, 2,764 papers were evaluated by the two HFOI section editors in the pre-selection phase based on title, keywords, and abstract. Eighty-seven papers were preselected. The list was further refined to 15 papers, which were carefully evaluated 
by 4 external reviewers. Reviewers used the standard evaluation grid of the Yearbook with a score from 0 to 100 using 5 criteria: (i) Significance (Topic's importance and Scientific and/or practical impact); (ii) Quality of scientific and/or technical content; (iii) Originality and innovativeness; (iv) Coverage of related literature; (v) Organization and clarity of presentation. The five best papers were selected in a discussion with the Yearbook editorial committee.

Pre-defined inclusion/exclusion criteria were applied during the pre-selection phase. We excluded: (a) papers focused on the evaluation of a precise system, with no general consideration on adoption issues (e.g. recommendations); (b) studies simply measuring user satisfaction; (c) studies only focused on the ergonomics or usability of systems or only dealing with human-computer interactions issues in a narrow sense; (d) studies relying on questionnaires without additional observational methods; (e) review papers. Qualitative studies focusing on the so-called "perception" or "acceptance" of HIT by end users were excluded if they focused on the evaluation of a particular system without generalizable observations. Studies of HIT interface usability were also excluded except when they modeled cognitive (e.g. perceptual or attentional) factors implied in the use of the system. We included studies: (a) making use of paradigms, methodologies, models, and empirical results from cognitive or social sciences; (b) explicitly adopting an HFOI approach to medical informatics; (c) discussing theoretical or methodological issues related to the HFOI approach; (d) using in situ field observation rather than questionnaires or semi-structured interviews. The main reason for (d) is that "direct observation is advantageous compared to other methods (e.g., interviews and focus groups) in studying phenomena [...] since the data are based on what providers are seen doing, not on what providers say they do, which can often be different" [21].

\section{Correspondence to:}

Gunnar Declerck

LIMICS, UMRS 1142

Centre de recherche des Cordeliers, Escalier D, 2e étage

15 , rue de l'école de médecine

75006 Paris, France

E-mail: gunnar.declerck@utc.fr

Appendix: Content Summary of Selected Best Papers for the 2014 Edition of the IMIA Yearbook, Section HFOI

\section{Babbott $S$, Manwell LB, Brown R, Montague E, Williams E, Schwartz M, Hess E, Linzer M}

Electronic medical records and physician

stress in primary care: results from the MEMO Study

\section{J Am Med Inform Assoc 2014 Feb;21(el):e100-6}

Using data from the 2001 to 2005 MEMO (Minimizing Error, Maximizing Outcome) study, the authors aimed to assess the relationships between the number of EHR functions, primary care work conditions, and physician stress, burnout, job satisfaction, and intent to leave the practice. Primary care physicians from inner city clinics, academic, and managed care clinics, and small rural clinics were recruited. A survey including qualitative scales was used to measure physician stress and satisfaction, and perception of time pressure. Clinic managers also completed a questionnaire querying office characteristics including the presence of an EHR system, its features, and the number of years since its initial deployment. Clinics were classified depending on whether their EHR system had a high $(50 \%$ of the clinic sample), moderate $(24 \%)$, or low (26\%) number of functions.

No clear association was observed between the number of years since EHR implementation and work stress. Two important findings are highlighted by the authors: (a) physicians using EHRs with a moderate number of functions report more stress and less job satisfaction than physicians using EHRs with a low number of functions; (b) while job stress decreases modestly for physicians with sophisticated systems as compared to physicians using an EHR with a moderate number of functions, time pressures during examinations and office visits are more related to adverse physician outcomes in the high EHR function cluster. One possible explanation of (a) is that physicians using the moderate functionality EHR systems have to use a hybrid paper/electronic system, which is demanding in terms of cognitive resources. Physicians have to use computers to view results, but switch to paper for prescription. The result (b) suggests that using highly sophisticated EHR systems may be particularly challenging when the time allotted is lower than the time necessary to provide quality care, forcing clinicians to act quickly during the patient visit. To mitigate the potential negative effects of practice transformation, the authors recommend developing transition strategies that prepare offices for increased stress and mitigate its effect, such as regular optimization meetings coupled with a responsive information technology group.

Flanagan ME, Saleem JJ, Millitello LG, Russ $A L$, Doebbeling BN

Paper- and computer-based workarounds to electronic health record use at three benchmark institutions

\section{J Am Med Inform Assoc 2013 Jun;20(el):e59-66}

Current EHR systems do not always perform optimally; they may not support all aspects of the clinical work and may necessitate (sometimes significant) changes to clinical work practices or to professional responsibilities. HPs may perceive EHRs as impediments to delivering efficient care, resulting in the development of workarounds (i.e. non-standard procedures that do not follow explicit or implicit rules, assumptions, workflow regulations, or intentions of system designers) to manage efficiency and meet specific information and task requirements. Using rapid ethnographic direct observation methods across 3 healthcare organizations, the purpose of this study was to identify and classify the types of workarounds used by HPs, and to understand the conditions and work processes surrounding these workarounds.

Consistent with prior research, 11 workaround categories (whether paper- or computer-based or both) were identified. The most widespread and noteworthy are the following: efficiency workarounds, i.e. using a workflow process that improves actual or perceived efficiency, such as 
paper notes with vitals and responses to screening questions for later data entry, or copying and pasting text from previous EHR patient progress notes into a new note; awareness workarounds, used for notifying or alerting a co-worker of new or important information, e.g. making notes on paper-based patient encounter forms to notify a provider about patient information such as vitals at in-take; memory workarounds, i.e. actions to remind about relevant information, such as making paper notes for completing a task later (e.g. finish unsigned progress notes) or to follow-up on a task (e.g. administer a shot); trust-related workarounds, i.e. actions resulting from greater trust in paper over electronic tools, such as writing down a summary of a given patient's chief complaint in case the EHR is not available when the patient arrives; "no correct path" workarounds, which are developed when a desired option for completing a task does not exist in the EHR system, e.g. giving partial doses of medications but documenting that the full dose was administered.

Some interesting claims raised by the authors should be highlighted: (a) most workaround categories were present across the different institutions, which suggests some consistent weaknesses in EHR systems; (b) low-tech media such as paper notes and white boards have powerful advantages that cannot be easily replicated electronically: they are very efficient and adaptable (e.g. "users can quickly and easily create prospective memory aids for non-routine events, reconfigure data to highlight elements that are important in a specific context, or track data over time that may become important later in solving a specific problem") and, importantly, they have an intrinsically transitory quality (as soon as they do not turn out to be useful, they can be abandoned). "It might be unrealistic to expect that EHRs will ever eliminate the need for user-created, paper-based cognitive support"; (c) workarounds must be considered as a system-level issue, involving people, technology, context, and other organizational factors and interactions among these factors, and not as the result of non-compliance or technology-resistance of HPs.
Hilligoss B, Zheng $\mathrm{K}$

Chart biopsy: an emerging medical practice enabled by electronic health records and its impacts on emergency department-inpatient admission handoffs

\section{J Am Med Inform Assoc 2013 Mar- Apr;20(2):260-7}

Drawing on qualitative data (including semi-structured interviews, observations, and conversations records) from a 2-year ethnographic study in an emergency department (ED), this study aimed at examining (a) how the use of an inpatient EHR system empowers physicians receiving admission handoffs to be better informed about patient cases and thus better positioned in handoff conversations, and (b) how the improved access to patient data enabled by EHRs creates new possibilities of information use and reuse in healthcare settings, possibly making clinical practice safer, more efficient, and more effective.

The study's most valuable contribution is the identification and analysis of an emerging EHR-enabled practice, the so-called pre-handoff "chart biopsy". Chart biopsy is generally performed by inpatient physicians prior to taking admission handoffs, and involves the targeted selection of information and data from the EHR to aid in the process of learning about the patient during the discussion with the ED physician. This process has at least three functions: (1) obtaining an overview of the patient (a thorough understanding is not possible because of time constraints); (2) preparing for handoff and subsequent care, anticipating patient needs, and fulfilling documentation requirements; it also prepares inpatient physicians to assume responsibility and begin planning care; (3) defending against potential biases: rather than simply accepting patients as presented in handoffs, inpatient physicians use chart biopsy to reexamine the ED physician's understanding of the patient and to guard against biases such as diagnosis momentum, i.e. the tendency to establish diagnosis without adequate evidence.

As illustrated by the emerging chart biopsy practice, EHRs open up new possibilities of information reuse by enabling more convenient access to more diverse informa- tion, which can impact important clinical processes and outcomes. "The understanding constructed during a chart biopsy enables the inpatient physician to listen, think, and interact more critically during the handoff, including asking more meaningful questions and proposing alternative understandings of the patient's case. [The physicians] enter handoff not as passive recipients of information, but rather as active co-constructors of an understanding of the patient and as active participants in the planning of care."

The authors discuss the implications on the design and the evaluation of EHR systems, stressing the importance of minimizing information fragmentation, a common issue in current EHR design. Fragmentation necessitates significant navigation efforts in order to assemble data from discrete screens and documents in order to construct an understanding of the patient and may thus hinder the chart biopsy process.

\section{Lanham HJ, Sittig DF, Leykum LK, Parch- man ML, Pugh JA, McDaniel RR Understanding differences in electronic health record (EHR) use: linking individual physicians' perceptions of uncertainty and EHR use patterns in ambulatory care}

\section{J Am Med Inform Assoc 2014 Jan $1 ; 21(1): 73-81$}

Drawing on complexity science for a theoretical framework and on the medical and organizational literature on physicians' perception and attitude towards uncertainty, this qualitative study aimed to assess if the way physicians use EHRs is associated with their responses to uncertainty and to identify new potentially important variables in explaining differences in EHR use patterns. Semi-structured interviews using an ethnographic approach (focusing on physicians' experiences and beliefs about using an EHR, and perceptions of how it influences their interaction with patients) and the direct observation of physicians $(\mathrm{N}=28)$ working in a multispecialty outpatient care organization were realized.

Three categories of attitudes towards uncertainty and strategies for managing uncertainty were identified: (a) uncertainty reduction: reducing uncertainty and dimin- 
ishing knowable risk through information gathering (e.g. looking up a test result in an EHR) or information processing; the limit of this strategy is that the requested information must be available; (b) uncertainty absorption: assimilating or incorporating uncertainty into local circumstances through argumentation, narration, and information exchanges with others (physicians, nursing staff, patients, etc.): through these exchanges, new meaning is inferred and uncertainty is absorbed. This strategy is used for managing uncertainties in healthcare delivery that are irreducible or not resolvable with information (e.g. how a patient will respond to a medication); (c) hybrid strategy: combination of the uncertainty reduction and absorption perspectives. Physicians' use of EHR was categorized as high (16 physicians), medium (9), or low (3) based on factors such as the degree of EHR feature use, the level of EHR-enabled communication with others, and the frequency with which EHR use patterns changed.

Comparisons across physicians revealed a marked association between perceptions of uncertainty and EHR use patterns: (a) uncertainty reductionists tended to exhibit high levels of EHR use (13/13). They expressed the belief that the information contained in the EHR is paramount for patient care and they sought to reduce uncertainty by continually gathering, manipulating, and inputting information in the medical record; (b) uncertainty absorbers tended to exhibit low levels of EHR use (3/5). They expressed the belief that the ongoing and attentive exchange of information and the co-creation of knowledge during patient encounters are paramount for the care process, and that information in the EHR is often of limited use for patient care; (c) physicians with hybrid perspectives tended to exhibit medium levels of EHR use (8/10). They considered the information captured in the EHR as critical for their work and considered at the same time that information exchange with patients was a critical part of their practice. These findings suggest that perception and strategies to manage uncertainty are potentially important factors to understand the differences in EHR use patterns across physicians. These finding should be considered in EHR future design, implementation, and training efforts considering that current EHR systems "are generally designed to help clinicians manage the reducible uncertainty they face in caring for patients. Improvements can be made $[\ldots]$ in helping them manage the irreducible uncertainty they face."

\section{Smith SW, Koppel R \\ Healthcare information technology's rela- tivity problems: a typology of how patients' physical reality, clinicians' mental models, and healthcare information technology differ}

\section{J Am Med Inform Assoc 2014 Jan $1 ; 21(1): 117-31$}

The purpose of this strikingly original study was to model (a) patients" "concrete" - so to say - reality (RW for real world), (b) how clinicians represent this reality, i.e. their mental models (MM) of patients' conditions, and (c) how this reality and these mental models are embodied and formalized in EHRs (IT). The goal was to highlight inconsistencies, misunderstandings, and distortions among these three complex representations, and to serve as a tool for quality assessment and improvement of EHRs.

Based on several sources including literature, direct observations, hospital logs, personal communications, implementation teams' reports, and HIT vendor forums, the authors identified 45 misalignment scenarios classified in 5 general categories: (i) too coarse: the granularity of the EHR system language is insufficient to express some different RW and MM scenarios, which are wrongly mapped to the same element in the EHR, losing significant distinction. For instance, EHRs often fail to let the clinician enter age in days for newborn babies, or some units of measurement may be missing. Another example is a medication order that is not yet visible in the EHR because it has not been approved by the pharmacy; (ii) too fine: some RW scenarios distinctions are irrelevant and hence map to the same elements in MM, but the EHR system maps these scenarios into distinct elements in IT, thus creating an irrelevant distinction - e.g. an IT can have several separate records corresponding to the same patient because the name of this patient has been entered in different ways, or the IT considers that several medicines were delivered to the patient because the same barcode was unintentionally scanned several times; (iii) missing reality: the language of the EHR system fails to include some relevant parts or critical details of RW and MM - even though mapping between IT and MM may be articulated, it only covers a proper subset of the relevant mental models, for example, unreadable patient barcode wristbands for medication administration, or EHR predefined data lists with missing values; (iv) multiplicity: local user cultures (MM) apply implicitly understood distortions to their use of the system language (IT), which causes users who do not share that understanding to draw significantly incorrect conclusions about the underlying reality $(\mathrm{RW})-$ e.g. intentional understatement or overstatement of some diagnosis (because of legal, ethical, or bureaucratic issues) by the physicians of a given institution will be interpreted literally, and thus wrongly, by the physicians of another institution because they are not familiar with this local practice; (v) information distorted by iterative reflections among clinicians and IT systems: scenarios significant to clinicians' $\mathrm{MM}$ are represented in IT, but when the representation maps back to reality, it becomes significantly distorted, as it has passed through repeated iterations within IT, and between users and IT - e.g. copy-paste-induced errors or perceived unreliability of EHR systems with a history of failing at critical moments or because of frequent patches (such as too frequent changes in drug-drug interaction or dosage alerts, leading physicians to feel that things just appear and disappear without any understandable reason).

The impressive typology proposed in this article is a potentially very powerful tool for analyzing the misalignments between EHR representations and functionalities, and the information that is relevant for the care process, and for reducing these mismatches.

\section{References}

1. Health IT Dashboard, Office of the National Coordinator for Health IT (ONC), U.S. Department of Health and Human Services (HHS). http:// dashboard.healthit.gov/

2. Nambisan P, Kreps GL, Polit S. Understanding Electronic Medical Record adoption in the United 
States: Communication and sociocultural perspectives. Interact J Med Res 2013;2(1):e5.

3. Wright A, Henkin S, Feblowitz J, McCoy AB, Bates DW, Sittig DF. Early results of the meaningful use program for electronic health records. N Engl J Med 2013;368(8):779-80.

4. DesRoches CM, Worzala C, Bates S. Some hospitals are falling behind in meeting 'meaningful use' criteria and could be vulnerable to penalties in 2015. Health Aff 2013;32(8):1355-60.

5. Le coût du dossier médical personnel depuis sa mise en place. [The cost of personal health record since its establishment.];Cour des comptes 2012 (in French). http://www.ccomptes.fr/Publications/ Publications/Le-cout-du-dossier-medical-personnel-depuis-sa-mise-en-place.

6. Direction générale de l'offre de soins (DGOS). Programme Hôpital numérique. Rapport d'activité 2013. http://www.sante.gouv.fr/le-programme-hopital-numerique.html.

7. Black AD, Car J, Pagliari C, Anandan C, Cresswell K, Bokun T, McKinstry B, Procter R, Majeed A, Sheikh A. The impact of eHealth on the quality and safety of health care: a systematic overview. PLoS Medicine 2011;8(1):e1000387.

8. DesRoches CM, Campbell EG, Vogeli C, Zheng J, Rao SR, Shields AE, et al. Electronic health records' limited successes suggest more targeted uses. Health Aff 2010;29(4):639-46.

9. Bowman S. Impact of Electronic Health Record Systems on Information Integrity: Quality and Safety Implications. Perspect Health Inf Manag 2013 Oct 1;10:1c.

10. American Health Information Management Association (AHIMA). Data Quality Management Model. Chicago, IL: AHIMA; 2012.

11. Balka E, Tolar M, Coates S, Whitehouse S. Socio-technical issues and challenges in implementing safe patient handovers: Insights from ethnographic case studies. Int J Med Inform 2013;82(12):e345-e357.

12. Jenkin A, Abelson-Mitchell N, Cooper S. Patient handover: time for a change? Accid Emerg Nurs 2007:15:141-7.

13. Wong MC, Turner P, Yee KC. Socio-cultural issues and patient safety: a case study into the development of an electronic support tool for clinical handover. Stud Health Technol Inform 2007:130:279-89.

14. Friedberg MW, Chen PG, Van Busum KR, Aunon F, Pham C, Caloyeras J, et al. Factors Affecting Physician Professional Satisfaction and Their
Implications for Patient Care, Health Systems, and Health Policy. Rand Corporation; 2013.

15. Beasley JW, Wetterneck TB, Temte J, Lapin JA, Smith P, Rivera-Rodriguez AJ, et al. Information chaos in primary care: Implications for physician performance and patient safety. J Am Board Fam Med 2011 Nov-Dec;24(6):745-51.

16. Showell C, Thomas M, Wong MC, Yee KC, Miller $\mathrm{S}$, Pirone C, Turner P. Patient safety and sociotechnical considerations for electronic handover tools in an Australian e-health landscape. Stud Health Technol Inform 2010;157:193-8.

17. O'Malley AS, Grossman JM, Cohen GR, Kemper NM, Pham HH. Are electronic medical records helpful for care coordination? Experiences of physician practices. J Gen Intern Med 2010;25(3):177-85.

18. Holden RJ. Cognitive performance-altering effects of electronic medical records: an application of the human factors paradigm for patient safety. Cogn Technol Work 2011;13(1):11-29

19. Samaan ZM, Klein MD, Mansour MD, DeWitt TG. The impact of the electronic health record on an academic pediatric primary care center. J Ambul Care Manage 2009;32(3):180-7.

20. Babbott S, Manwell LB, Brown R, Montague E, Williams E, Schwartz M, et al. Electronic medical records and physician stress in primary care: results from the MEMO Study. J Am Med Inform Assoc 2014;21(e1):e100-e106.

21. Flanagan ME, Saleem JJ, Millitello LG, Russ AL, Doebbeling BN. Paper-and computer-based workarounds to electronic health record use at three benchmark institutions. J Am Med Inform Assoc 2013;20(e1):e59-e66.

22. Wears RL, Perry SJ. Shadows and ghosts: the role of hidden information in a US healthcare setting. Proceedings of the International Ergonomics Association 2006 Congress. Maastricht, Netherlands: International Ergonomics Association; 2006.

23. Wears RL. The chart is dead-long live the chart. Ann Emerg Med 2008;52(4):390-1.

24. Buntin MB, Burke MF, Hoaglin MC, Blumenthal D. The benefits of health information technology: a review of the recent literature shows predominantly positive results. Health Aff 2011;30(3):464-71.

25. Turner AM, Reeder B, Ramey J. Scenarios, personas and user stories: User-centered evidence-based design representations of communicable disease investigations. J Biomed Inform 2013;46(4):575-84.

26. Mathews SC, Pronovost PJ. The need for systems integration in health care. J Am Med Inform Assoc
2011;305(9):934-5

27. Garfinkel H. Studies in ethnomethodology. NJ: Englewood Cliffs; 1967.

28. Militello LG, Arbuckle NB, Saleem JJ, Patterson E, Flanagan M, Haggstrom D, et al. Sources of variation in primary care clinical workflow: Implications for the design of cognitive support. Health Informatics J 2014 Mar;20(1):35-49.

29. Berg M. Patient care information systems and health care work: a sociotechnical approach. Int J Med Inform 1999;55(2):87-101.

30. Norman DA. Things that make us smart: Defending human attributes in the age of the machine. Basic Books (AZ);1993.

31. Hutchins E. How a cockpit remembers its speeds. Cognitive science 1995;19(3);265-88.

32. Hollan J, Hutchins E, Kirsh D. Distributed cognition: toward a new foundation for human-computer interaction research. ACM Trans Comput Hum Interact 2000;7(2):174-96.

33. Zhang J, Patel VL. Distributed cognition, representation, and affordance. Pragmatics \& Cognition 2006;14(2):333-41

34. Norman DA. Cognitive artifacts. Department of Cognitive Science, University of California, San Diego; 1990.

35. Kirsh D. The intelligent use of space. Artif Intell 1995;73(1):31-68.

36. Lave J. Cognition in practice: Mind, mathematics and culture in everyday life. Cambridge University Press; 1988.

37. Gibson JJ. The ecological approach to visual perception. Psychology Press; 1986.

38. Varela FJ, Thompson ET, Rosch E. The embodied mind: Cognitive science and human experience. MIT press; 1991

39. Lakoff G, Johnson M. Philosophy in the flesh: The embodied mind and its challenge to western thought. Basic Books (AZ); 1999.

40. Clark A, Chalmers D. The extended mind. Analysis 1998;58(1):7-19.

41. Goody J. The domestication of the savage mind. Cambridge; New York: Cambridge University Press; 1977.

42. Park SY, Lee SY, Chen Y. The effects of EMR deployment on doctors' work practices: A qualitative study in the emergency department of a teaching hospital. Int J Med Inform 2012;81(3):204-17.

43. Gille B. The History of Techniques. New York: Gordon and Breach Science Publishers; 1986.

44. Schuemie MJ, Talmon JL, Moorman PW, Kors JA. Mapping the domain of medical informatics. Methods Inf Med 2009;48(1):76. 